\title{
A clinical analysis and diagnosis course of children with PURA-related neurodevelopmental disorders in Tianjin, China
}

\author{
Wenshuang Zhang ( $\sim$ zws19702004@sina.com ) \\ Tianjin children's Hospital \\ Yanan Li \\ Tianjin Children's Hpspital
}

\section{Research Article}

Keywords: diagnosis course, PURA-related, neurodevelopmental disorders, c.154dupG (p.L54Afs*147), followup

Posted Date: April 21st, 2021

DOI: https://doi.org/10.21203/rs.3.rs-445079/v1

License: (a) (i) This work is licensed under a Creative Commons Attribution 4.0 International License. Read Full License 


\section{Abstract}

PURArelated neurodevelopmental disorders are characterised by moderate to severe neurodevelopmental delay, and their morbidity rate is clinically low in children. We retrospectively conducted a clinical analysis and diagnosis course of neurodevelopmental disorders and found it was related to PURA by whole-exome sequencing (WES). A 35-day male infant was found to have mutations in the PURA gene, and the clinical manifestations were laryngeal sputum, feeding difficulties, insufficient power during crying, high tension, hypothermia (the highest anal temperature monitored was only $37.2^{\circ} \mathrm{C}$ ), poor circulation, postnatal weight loss (abnormal weight loss) and apnoea. WES found mutations for c.154dupG (p.L54Afs*147), which caused frameshift mutations of the amino acid, resulting in a preliminary diagnosis of pathogenic mutation on a site that has not been reported before.

Conclusions: The clinical manifestations of PURA gene mutations lack specificity, which are always manifested as multiple system involvement. Gene detection is helpful to improve the level of clinical diagnosis. According to the literature review, the disease onset age was mostly less than 18 years old, and each system is affected. At present, the treatment measures for this gene mutation and its long-term prognosis are unclear. However, to date, the maximum follow-up can reach 27 years. No literature suggests that the clinical phenotype and prognosis are related to genotype, indicating that the prognosis can be improved with appropriate symptomatic treatment and surgical intervention.

What is known:

PURA-related neurodevelopmental disorders are a rare variety of autosomal dominant diseases, which include PURA syndrome and 5q31.3 deletion syndrome. Both are characterised by moderate to severe neurodevelopmental delay and other nonspecific clinical manifestations.

What is new:

1.No more than 100 cases of neurodevelopmental disorders associated with PURA have been reported to date.

2.This work is the first time a complete case has been followed for such a long time period.

3. The site of c.154dupg (p.L54Afs*147) of the PURA gene has not been reported to date.

\section{Introduction}

Purine-rich element binding protein A (PURA)-related neurodevelopmental disorders include PURA syndrome, caused by a heterozygous pathogenic sequence variant in PURA, and $5 \mathrm{q} 31.3$ deletion syndrome, caused by a genomic 5q31.3 deletion encompassing all or part of the PURA gene. In China, the reported cases do not exceed 10 cases, with a clear diagnosis of PURA syndrome only in two cases. both of them are term infants,and had the same locus of variation. At 4 days after the onset age, one patient died, whereas the other died at 15 days after the onset age. Children were followed up for half a year, and findings showed that the PURA syndrome still existed, as evidenced by breathing and feeding difficulties, low muscle tone, 
psychomotor retardation and other symptoms. The child described in this paper is the latest onset of illness in China. The PURA syndrome is difficult to diagnose based solely on clinical manifestations; its incidence rate amongst children is rare and has been described only in several individuals to date. This paper presents one case.

\section{A Clinical Analysis And Diagnosis Course}

A 35-day-old male baby was admitted to the outpatient clinic of Tianjin Children's Hospital (Tianjin, China) at 14:00 in February. The patient had been found with laryngeal stridor for 2 days and feeding intolerance for 1 day. The family members stated that the child had been crying less and moving less, and he had lost $0.6 \mathrm{~kg}$ of weight after birth. The baby was born via full-term vaginal delivery, G1P1 and had no other evidence of major medical or birth defects. The mother of the baby was naturally pregnant and had fever on the 2nd day after giving birth. The blood culture in the hospital showed Escherichia coli and enterococcus infection. She was suspectedly diagnosed with sepsis in the local hospital and discharged after 15 days of antiinfection with meropenem. The parents were not engaged in toxic or harmful work and had no history of diabetes, epilepsy, neurological deafness, other family genetic illness and inbreeding. The following results were obtained by physical examination after admission: anal temperature, $37.2{ }^{\circ} \mathrm{C}$; pulse, $130 / \mathrm{min}$; respiration, $21 / \mathrm{min}$; blood pressure, $80 / 50 \mathrm{mmHg}(1$ $\mathrm{mmHg}=0.133 \mathrm{kPa}$ ); body weight, $3.4 \mathrm{~kg}$; head circumference, $34 \mathrm{~cm}$; body length, $53 \mathrm{~cm}$; clear consciousness; poor response; irregular respiration rate; and moist rales in both lungs. Muscle tone was low, crying was absent and movement was minimal. On admission, the white blood cell count was $20.22 \times 10^{9} / \mathrm{L}$, neutrophil was $56.2 \%$, monocyte was $10.1 \%$, platelet was $216 \times 10^{9} / \mathrm{L}$, haemoglobin was $149 \mathrm{~g} / \mathrm{L}$ and c-reactive protein (CRP) was $25.3 \mathrm{mg} / \mathrm{L}$. Arterial blood gas was normal. Chest film showed bronchopneumonia. The child was admitted with a diagnosis of bronchopneumonia.

Given the severity of the illness in the child, he was admitted to the intensive care unit, where given oxygen atomisation and empirical application of $60 \mathrm{mg} / \mathrm{kg} /$ day cephalosporin was conducted for anti-infection treatment.But at $2 \mathrm{~h}$ after admission, the patient suddenly stopped breathing, and his blood oxygen saturation level was only $78 \%$. Timely rescue support was provided via tracheal intubation and mechanical ventilation of symptoms. The child had a rectal temperature of $35.7{ }^{\circ} \mathrm{C}$, breathing rate of 20 times/min, irregular breathing rhythm, pulse of $95 \mathrm{bpm}$, transcutaneous oxygen saturation of $90 \%$, oxygen flow of $4 \mathrm{~L} / \mathrm{min}$, bad spirit and exhibited lethargy and cool peripheral limbs. As a result of the child's poor general condition on admission, the neonatal behavioural assessment scale (NABA, a test in China that can help determine newborns' ability to behave and detect minor brain injuries early, allowing for early intervention to prevent and treat disability) was not appropriate.

On the basis of the significant increase in the inflammatory index after admission, such as peripheral blood white blood cells (mainly neutral), CRP, primitive calcitonin and the mother's postpartum history of suspected sepsis, the possibility of drug-resistant bacterial infection should be considered, so the antibiotic meropenem combined with linezolid anti-infection 
treatment was adjusted. After oxygen inhalation, physical heating, atomisation, anti-infection and other corresponding treatment measures, the blood oxygen saturation on the 3rd day after admission was better than before and rose to $99 \%$ without oxygen inhalation, but the child still demonstrated irregular breathing. On the 5th day after admission, we performed lumbar puncture examination, and the cerebrospinal fluid biochemistry was generally normal. At this time, the blood routine examination showed that the inflammatory indexes decreased, and linezolid was discontinued. The re-examination of arterial blood gas showed pH 7.40, partial pressure of carbon dioxide of $52.4 \mathrm{mmHg}$, partial pressure of oxygen of $110 \mathrm{mmHg}$, residual alkali of $6.4 \mathrm{mmol} / \mathrm{L}$ and actual bicarbonate of $32.5 \mathrm{mmol} / \mathrm{L}$. These findings indicated compensatory respiratory acidosis combined with metabolic alkalosis, and subsequent observation was necessary. On the 7th day of admission from the machine, the child's oxygen saturation reached $98 \%$, but the rectal temperature was sustained at $35.7{ }^{\circ} \mathrm{C}-37.3^{\circ} \mathrm{C}$ and the breathing rate was 20-30 times/min. Moreover, the child demonstrated poor sucking ability and low limb muscle tension. At 21 days in the hospital, the child's systemic symptoms showed an improvement; the milk feeding rate was $60 \mathrm{~mL} / \mathrm{s}, \mathrm{Q} 3 \mathrm{H}$ and daily calorie intake was about $76.2 \mathrm{kcal} / \mathrm{kg} /$ day. The NABA was performed on the child at discharge (Table 1).

Table 1. Neonatal 20-item Behavioural Neurological assessment (NABA) at discharge from the hospital in this case 


\begin{tabular}{|c|c|c|c|c|c|}
\hline \multirow{2}{*}{\multicolumn{2}{|c|}{ ITEM }} & \multicolumn{3}{|c|}{ SCORE } & \multirow[b]{2}{*}{ To } \\
\hline & & 0 & 1 & 2 & \\
\hline \multirow[t]{6}{*}{$\begin{array}{c}\text { Behavioral } \\
\text { capacity }\end{array}$} & $\begin{array}{l}\text { 1. formation of } \\
\text { sound habits }\end{array}$ & $>11$ & $7-10$ & $\leq 6$ & 2 \\
\hline & $\begin{array}{l}\text { 2. the formation of } \\
\text { light habits }\end{array}$ & $>12$ & $7-11$ & $\leq 7$ & 1 \\
\hline & 3. responding to cluck & The head and & Eye or head turning $<60^{\circ}$ & Eye or head turning $\geq 60^{\circ}$ & 2 \\
\hline & 4. responding to face talk & Idem & Idem & Idem & 2 \\
\hline & 5.reaction to the red ball & Idem & Idem & Idem & 1 \\
\hline & 6. comfort & can't & Difficulties & Easy or automatic & 2 \\
\hline \multirow{4}{*}{$\begin{array}{l}\text { Passive } \\
\text { muscle } \\
\text { tension }\end{array}$} & 7. scarf levy & $\begin{array}{l}\text { Around the } \\
\text { neck }\end{array}$ & Elbow slightly midline & Elbow not in line & 2 \\
\hline & 8. forearm bounce back & No & Slow Weak $>8$ & Active Repeat $<3$ & 2 \\
\hline & 9. popliteal horn & $>110^{\circ}$ & $100-110^{\circ}$ & $\leq 90^{\circ}$ & 1 \\
\hline & 10. Lower limb bounce & No & Slow, weak & Active repeat able & 1 \\
\hline \multirow{4}{*}{$\begin{array}{l}\text { Active } \\
\text { muscle } \\
\text { tension }\end{array}$} & 11. Head erect & Missing or & Difficulties & Okay, head and body on & 2 \\
\hline & 12. Holding hands & No & Weak & Okay, repeatable & 2 \\
\hline & 13. Pull reaction & No & Lift up parts of the body & Bring up the whole body & 1 \\
\hline & 14. Supporting responses & No & Incomplete, short & Strong an supportiv & 1 \\
\hline \multirow{3}{*}{$\begin{array}{l}\text { Original } \\
\text { reflection }\end{array}$} & 15. Step or placement & No & Lead to difficulties & Okay, repeatable & 1 \\
\hline & 16. Embrace & No & Weak, incomplete & Okay, totally & 2 \\
\hline & 17. Sucking & No & Weak & Okay, synchronized & 2 \\
\hline \multirow{3}{*}{$\begin{array}{c}\text { General } \\
\text { assessmen } t\end{array}$} & 18. Awareness & coma & Sleepiness & Normal & 1 \\
\hline & 19. Crying & No & Weak or excessive & Normal & 1 \\
\hline & 20. Degree of activity & Lack or & Slightly & Normal & 1 \\
\hline \multicolumn{5}{|c|}{ Total } & $\underline{3}$ \\
\hline
\end{tabular}

During admission, the head MRI of the child showed patchiness and slightly longer T1 and T2 signal shadow in the bilateral frontal and parietal ventricles, a small amount of haemorrhage in the subarachnoid space, widened extracerebral space, soft tissue swelling in the left frontal scalp and unorganised adjacent cranial plate (see Figure 1). Electroencephalogram (EEG) showed low to medium activity with amplitude of 1.5-2.5 Hzס in awake state and a few low-amplitude fast waves. The voltage of each conduction part was less than $20 \mu \mathrm{V}$. There was no epileptic discharge or abnormal EEG in awake state, and the voltage of each conduction part was low. 
Upon discharge, the family members were informed that 'pneumonia sepsis' could not fully explain the current clinical manifestations and physical examination of the child. After reviewing the literature, the child was suspected to be suffering from congenital or genetic diseases. After seeking the consent of the child's family members, the peripheral blood of the child and his parents was obtained for whole-exome sequencing (WES). The results of genetic testing showed that the child had variations in PURA gene C. 154dupG (p.L54Afs*147), while the blood samples of his parents showed no variation in this locus. The disease associated with this locus was autosomal dominant mental deficiency type 31 associated with the PURA gene. Gene detects: WES found a heterozygous mutation at the dupG site of Pura gene c.154, which changed leucine 54 into alanine, causing premature termination at 147 and frameshift mutation (Figure 2). At present, there are no studies about whether the variants disrupted the alleles of all protein translation, or mutations of codon translation if proteins are pathogenic. However, according to the American College of Medical Genetics and Genomics (ACMG) guidelines $^{(1)}$, it was determined to be pathogenic: 口PVS1 (very strong): this mutation is a frameshift mutation, which may lead to the loss of gene function; $\square$ PM2 (medium): the frequency in the normal population database is -, which is a low frequency variation; and $\square \mathrm{PM} 6$ (medium): a new variant that has not been verified by parental samples. According to the pathogenic mutation classification criteria: PVS1 (very strong) +PM2 (moderate) +PM6 (moderate), the mutation was determined to be pathogenic.

After the discharge of the child, at 1 month after the call to reply, the milk volume of the child was increased to $100 \mathrm{~mL} / \mathrm{q} 4 \mathrm{~h}$. The child's cry was louder than before, general movement increased and drowsiness was significantly better than before, but he still showed slow response to sound and demonstrated feeding difficulties, such as coughing when feeding and poor sucking. Subsequently, he received irregular follow-up in the outpatient department of genetics and metabolism and rehabilitation department of our hospital. One year later, the child's weight was $10.5 \mathrm{~kg}$. He was about $80 \mathrm{~cm}$ long; had strabismus and poor muscle strength; could not turn over; sat alone for not more than $1 \mathrm{~min}$; displayed irritability, slow sound response and poor speech development; could only recognise the mother; and did not know the rest of the family. The family did not complain of the child having irregular breathing patterns. The child's current diet comprised vegetables and meat.

\section{Discussion}

The human gene PURA is located at chromosome 5, band q31. This gene can encode PURa, a 322 amino

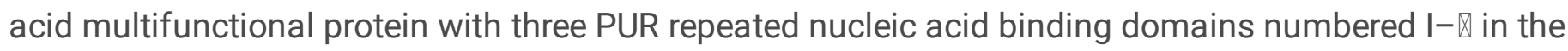
central region, and it is highly conserved from bacteria through humans ${ }^{(2,3)}$. The PUR domain consisting of PUR repeats I and II is the main nucleic-acid binding domain of this protein. PURa is also known to regulate expression of the myelin proteolipid protein Plp1, which is the predominant structural component of myelin sheaths in the central nervous system ${ }^{(4-6)}$.

The PURA gene is expressed everywhere: in the brain, muscles, heart and blood. In particular, the PURA gene is essential for brain development in mammals. The mice with knocked out PURA gene had developmental 
disorders in their brains and haematopoiesis and died shortly after birth ${ }^{(7)}$. This research suggested that PURA is required for postnatal development of the murine brain and is involved in both neuronal proliferation and maturation of dendrites ${ }^{(8)}$. PURA functions as a transcriptional activator, RNA transport protein in mRNA translation and regulator of DNA replication in the cell cycle ${ }^{(8-10)}$. As the PURA gene mutates, the DNA repair function is impaired, which can cause the degenerative changes of neurons, which may be related to the delayed neural development caused by the mutation of the PURA gene.

Pathogenic variants in PURA are associated with rare autosomal dominant disorders known as PURA-related neurodevelopmental disorders, which comprise the PURA syndrome and 5q31.3 deletion syndrome. Both are characterised by moderate to severe neurodevelopmental delay; most affected individuals are nonverbal, and many do not achieve independent ambulation ${ }^{(11)}$. Moreover, at least $50 \%$ of the patients report epilepsy in PURA-related neurodevelopmental disorders. In general, clinical symptoms usually begin with myoclonic seizures and develop into other types of epilepsy, including systemic rigidity matrix - twin seizure, tonic seizures and epileptic convulsion. Most attacks occur at neonatal age to 16 years old, and most patients $<5$ years old. The presence of a seizure or refractory epilepsy drug treatment is invalid. The disorder can be expressed on MRI in some individuals. MRI results should be consistent with the universality of brain white matter maturation disorder. The literature review showed that most of the subjects' brain exhibited white matter volume reduction, a slight increase in axial clearance outside the liquid, mild to moderate expansion, lateral ventricle head MRI contrast, subarachnoid bleeding and left frontal scalp soft tissue swelling. The prognosis of PURA gene-associated neurodevelopmental delay has not been clearly defined at home and abroad and requires long-term follow-up. A previous study reported one case of PURA syndrome in a child with epilepsy and PUR- $\triangle$ structure domain p.Phe233del mutation ${ }^{(12)}$. Several groups have now proposed that coding the PUR $\otimes$ repetitive sequence area may be more serious than coding the PURA gene mutation- $\bigotimes$ area

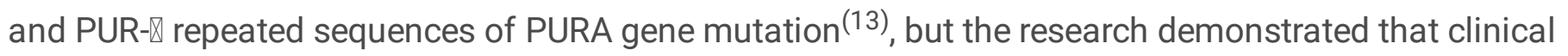
manifestation has no obvious relation with genotype ${ }^{(14)}$.

Notably, a significant phenotypic overlap was found between the PURA syndrome with the 5q31.3 microdeletion syndrome, the region within which PURA resides, suggesting that the loss of PURA in the microdeletion syndrome may be the cause of the overlapping phenotypes ${ }^{(15-16)}$. Clinical features are difficult to distinguish between these two diseases. Both are characterised by neurodevelopmental delays, learning disabilities, hypotonia, early feeding difficulties, speech and walking defects and seizure-like movements. One potential distinction, based on recent definitions, is that the PURA syndrome is diagnosed by a PURA pathogenic sequence variant in a proband (representing $90 \%$ of such PURA-related neurodevelopmental disorders). By contrast, the $5 q 31.3$ microdeletion syndrome is caused by a non-recurrent genomic $5 q 31.3$ deletion, which may encompass all or a part of PURA (10\% of affected individuals).

On the basis of the genetic test results of the baby, combined with his clinical manifestations of low tension, low temperature, feeding difficulties, increased hiccupping, irregular breathing and apnoea, the child was diagnosed with the PURA syndrome. Given his young age, the clinical manifestations of the neural function disorder were not found, but the child had a neonatal neurological score less than 35 points. This result indicated that the baby lacked nervous system development integrity. Long-term follow-up monitoring of the 
child's psychomotor development, eyesight, feeding difficulties, speech development of epileptic seizures or similar activities and skeletal motion complications (such as hip dysplasia and scoliosis) is necessary.

\section{Conclusion}

Clinically, children highly suspected of this disease should be closely observed, and their conditions should be tested as soon as possible. WES is currently the only diagnostic method available in the field. In terms of treatment, no specific treatment for this gene mutation presently exists. However, individuals often benefit when management is provided by a multidisciplinary team including relevant specialists. Corresponding symptomatic treatment measures should be actively adopted to improve the quality of life of children with this gene mutation after early detection.

\section{Declarations}

Conflict of interest The authors declare that they have no (financial) conflict of interest.

Ethical approval The case report was approved by the local ethical committee.

Informed consent The requirement of informed consent was waived for this retrospective study.

Source of support No sources of financial and material support to be declared.

Authors' contributions Wenshaung Zhang and Jing Chen contributed in the conception, analysis and interpretation of the study. Yanan Li drafted the manuscript for important intellectual content.

\section{References}

1. Richards, C.S., et al., ACMG recommendations for standards for interpretation and reporting of sequence variations: Revisions 2007. 2008.

2. Bergemann AD, Johnson EM. The HeLa Pur factor binds single-stranded DNA at a specific element conserved in gene flanking regions and origins of DNA replication.[J]. Molecular and Cellular Biology, 1992, 12(3):1257-1265.

3. Bergemann AD, Ma ZW, Johnson EM. Sequence of cDNA comprising the human pur gene and sequencespecific single-stranded-DNA-binding properties of the encoded protein. Molecular and Cellular Biology, 1992.

4. Graebsch A, Roche S, Niessing D. X-ray structure of Pur-a reveals a Whirly-like fold and an unusual nucleic-acid binding surface[J]. Proceedings of the National Academy of Sciences, 2009, 106(44):18521-18526.

5. Dobretsova A, Johnson JW, Jones RC, et al. Proteomic analysis of nuclear factors binding to an intronic enhancer in the myelin proteolipid protein gene[J]. Journal of Neurochemistry, 2010, 105(5):1979-1995.

6. Tanaka AJ, Bai R, Cho MT, et al. De novo mutations in PURA are associated with hypotonia and developmental delay[J]. Cold Spring Harbor Molecular Case Studies, 2015, 1(1):a000356. 
7. Khalili K, Valle LD, Muralidharan V, et al. Puralpha is essential for postnatal brain development and developmentally coupled cellular proliferation as revealed by genetic inactivation in the mouse. Molecular and Cellular Biology, 2003.

8. White MK, Johnson EM, Khalili K. Multiple roles for Pur-a in cellular and viral regulation[J]. Cell Cycle, 2009, 8(3):414-420.

9. Johnson EM. The Pur protein family: Clues to function from recent studies on cancer and AIDS[J]. Anticancer research, 2003, 23(3A):2093-2100.

10. Johnson EM, Chen PL, Krachmarov CP, et al. Association of human Pur alpha with the retinoblastoma protein, $\mathrm{Rb}$, regulates binding to the single-stranded DNA Pur alpha recognition element.[J]. Journal of Biological Chemistry, 1995, 270(41):24352-24360.

11. Reijnders, M., et al., PURA-Related Neurodevelopmental Disorders. 1993.

12. Cinquina $V$, Ciaccio $C$, Venturini $M$, et al. Expanding the PURA syndrome phenotype: A child with the recurrent PURA p.(Phe233del) pathogenic variant showing similarities with cutis laxa[J]. Molecular Genetics \& Genomic Medicine, 2020.

13. Hunt $D$, Leventer RJ, Simons $C$, et al. Whole exome sequencing in family trios reveals de novo mutations in PURA as a cause of severe neurodevelopmental delay and learning disability[J]. Journal of Medical Genetics, 2014, 51(12):806-13.

14. Reijnders $M$, Janowski R, Alvi M, et al. PURA syndrome: clinical delineation and genotype-phenotype study in 32 individuals with review of published literature.[J]. Journal of Medical Genetics, 2018, 55(2):104-113.

15. Hosoki K, Ohta T, Natsume J, et al. Clinical phenotype and candidate genes for the $5 q 31.3$ microdeletion syndrome[J]. American Journal of Medical Genetics Part A, 2012, 158A(8):1891-1896.

16. Boczek NJ, Macke E L, Kemppainen J, et al. Expansion of PURA -Related Phenotypes and Discovery of a Novel PURA Variant: A Case Report[J]. Child Neurology Open, 2020, 7:2329048X2095500.

\section{Figures}




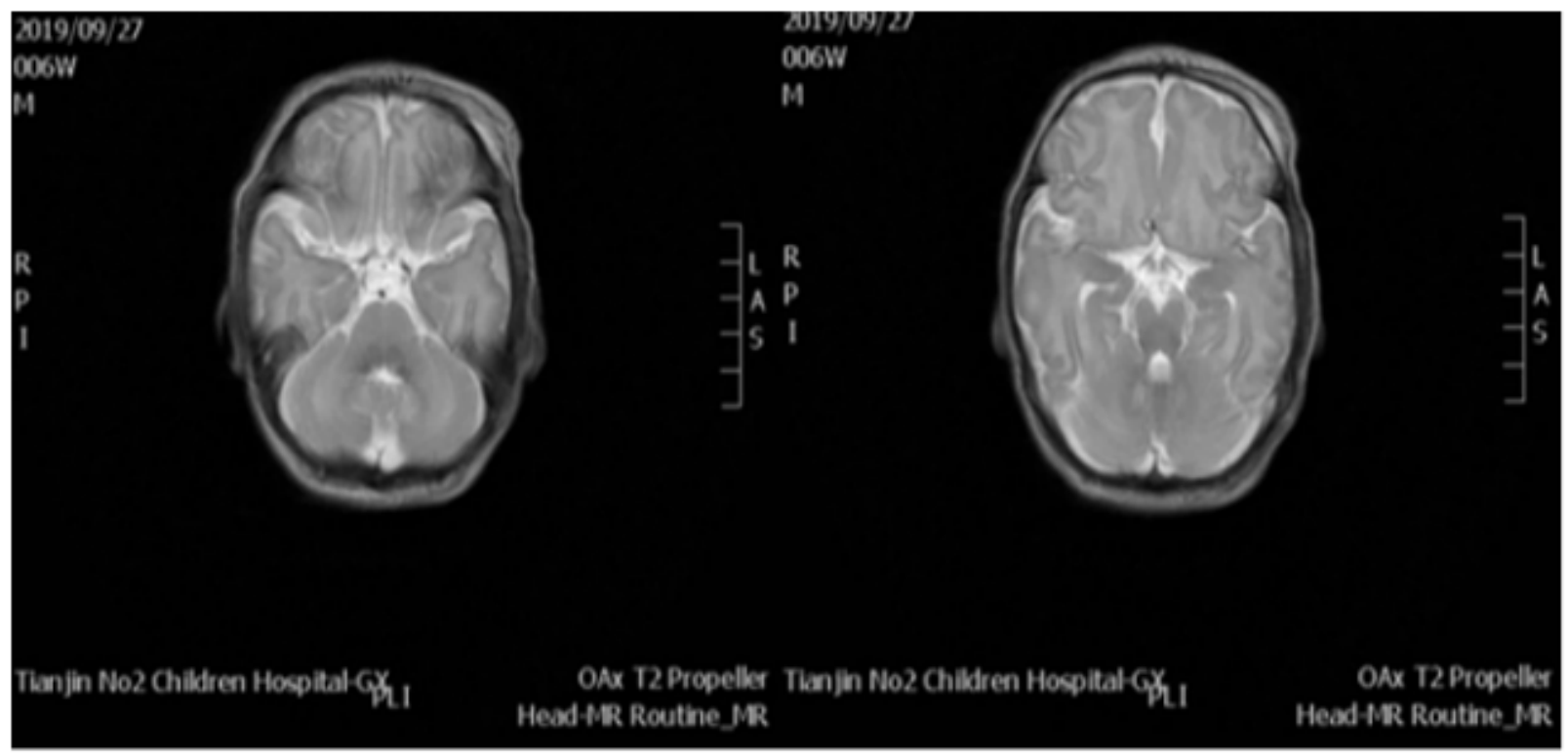

\section{Figure 1}

Brain MRI of the child, 43 days after birth

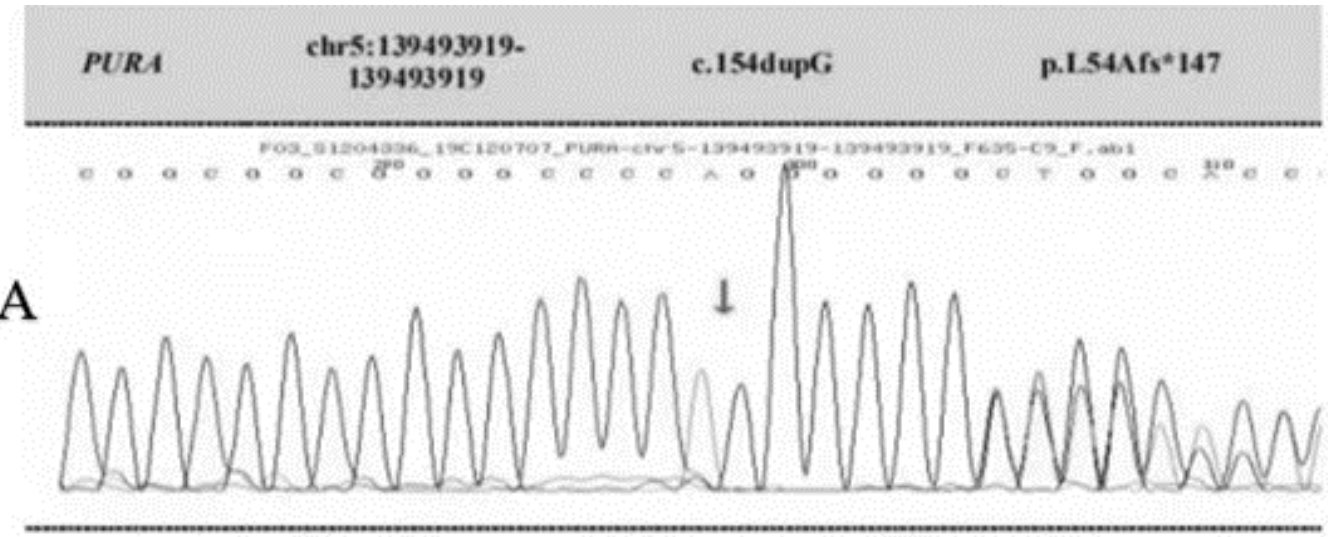

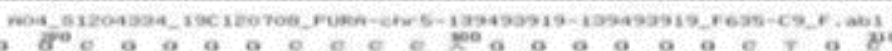

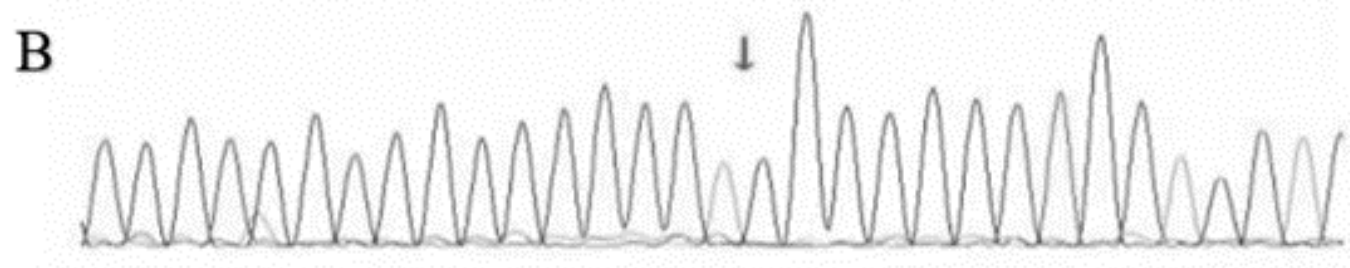

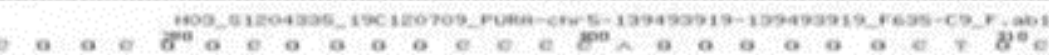

C

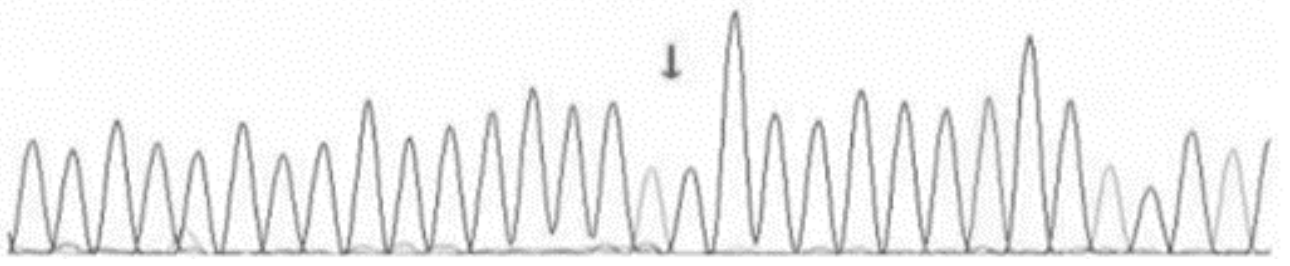

Figure 2 
Sanger sequencing of the C. 154dupG site of the PURA gene in the child and his parents. 